\title{
High Resolution Imaging of Virus in Liquid Droplets: The Application of Cryo- TEM Methodology to Improve Liquid-phase TEM Imaging of Biological Materials
}

\author{
Madeline J. Dukes $^{1}$, GM Jonaid ${ }^{2,3,4,5}$, William J. Dearnaley ${ }^{3,4,5}$, Michael A. Casasanta ${ }^{3,4,5}$ and Deborah \\ F. Kelly $3,4,5$ \\ ${ }^{1}$ Protochips, Inc. Morrisville, NC, USA. \\ ${ }^{2}$ Bioinformatics and Genomics Graduate Program, Huck Institutes of the Life Sciences, Pennsylvania \\ State University, University Park, PA, USA. \\ ${ }^{3}$ Department of Biomedical Engineering, Pennsylvania State University, University Park, PA, USA. \\ ${ }^{4}$ Materials Research Institute, Pennsylvania State University, University Park, PA, USA. \\ ${ }^{5}$ Center for Structural Oncology, Pennsylvania State University, University Park, PA 16802, USA.
}

In-situ environmental imaging which transforms the transmission electron microscope (TEM) into an active, nano-scale laboratory capable of conducting real-time experiments in non-vacuum environments, is an increasingly popular, albeit still challenging microscopy technique. Strategies to introduce and image samples in a liquid environment have led to a range of liquid-phase (LP)- TEM methods that enable researchers to safely observe the previously unseen nanoscale processes that dictate both material and biological mechanisms [1].

Highly electron scattering materials, such as metallic and core-shell nanoparticles have dominated LPTEM literature for the last decade due in no small part to their good electron scatter properties which make them considerably less challenging to visualize within a layer of liquid. However, the convergence of state-of-the-art commercial systems, low dose imaging techniques, improved data analysis and a more robust understanding of LP-TEM phenomena by the research community at large has enabled significant progress in imaging delicate, beam-sensitive soft materials such as micelles, polymers and biological species. In contrast, cryo-TEM, in which samples are flash frozen to preserve their structure during TEM imaging is overwhelmingly utilized with soft material samples, and as such, a wide range of low-dose and computational techniques have been developed in order to optimize the obtainable resolution [2]. Here we apply conventional 3D cryo-TEM imaging techniques to LP-TEM of adeno-associated virus (AAV) in thin layers of liquid.

AAV particles in liquid were imaged using a commercially available Protochips' Poseidon LP-TEM holder equipped with "microwell" E-chips [3]. These E-chips contain a series of microwells etched into the surface of the amorphous silicon nitride viewing membrane, as shown in Figure 1. This configuration allows minute quantities of liquid containing sample to be captured and isolated within the wells. In this case, the liquid is not continuously dispersed throughout the wells, but results in regions of varying liquid thickness between wells, capturing a variety of liquid states within a single experiment. Imaging was performed using $200 \mathrm{KV}$ TEM equipped with direct detector for low dose imaging. Liquid thickness regions were measured using energy filtered (EF) TEM as shown in Figure $2 \mathrm{a}$ and ranged from $52.5 \mathrm{~nm}$ (denoted as a thin liquid) to $105 \mathrm{~nm}$ (denoted as a thick liquid). A series of 20 second movies acquired using an electron flux of $1 \mathrm{e}^{-} \AA^{-2} \mathrm{~s}^{-1}$ and a frame rate of 40 frames per second (FPS) was acquired over multiple disparate regions within the viewable area of the liquid cell. We then applied conventional cryoTEM motion-correction algorithms, and advanced computing procedures to investigate the structure and dynamics of the AAV particles. Implementation of cryo-TEM computational techniques in combination with the low-dose, high sensitivity detector achieved a maximum resolution in our studies of 3.22 
angstrom for the AAV in liquid. More dynamically active AAV particles obtained a resolution of 7.24 angstrom (Figure 2C). This work demonstrates the exciting new possibilities of obtaining high resolution $3 \mathrm{D}$ reconstructions of biologically relevant species while preserving the innate dynamics that can be probed only in a liquid environment [4].

References:

[1] A Demmert et al, (2017) Visualizing Macromolecules in Liquid at the Nanoscale. In Liquid Cell Electron Microscopy, ed. F M Ross, pp 356. Cambridge, New York: Cambridge University Press.

[2] A Varano et al, Chemical Communications 51 (90) (2015), p. 16176.

[3] M Dukes et al, Microscopy and Microanalysis 20 (2014), p. 338.

[4] G Jonaid et al, Advanced Materials 2103221 (2021), In press
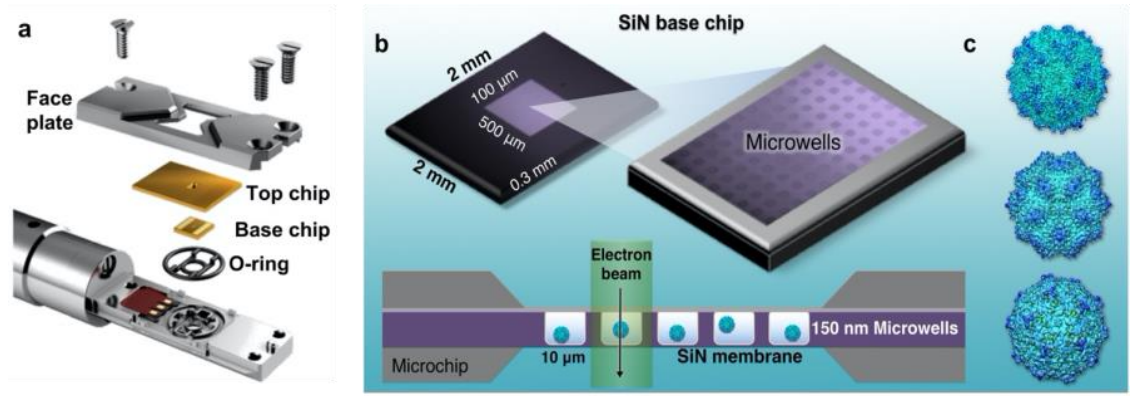

Figure 1. Example of the in-situ liquid-phase configuration used in imaging viral species in liquid. (a) Diagram of the Protochips' Poseidon in-situ liquid holder (b) Schematic of the microwell E-chip containing trapped droplets of sample. (c) Representative 3D structures of the AAV virus imaged in this study.
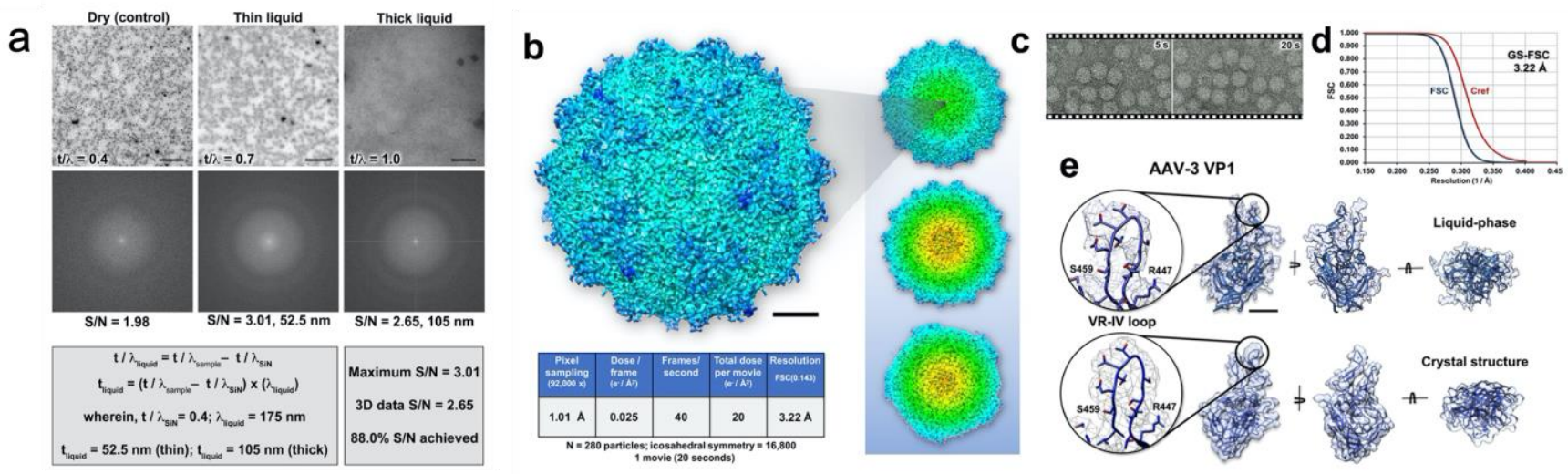

Figure 2. Examples and calculations for different liquid thickness regions (a) Liquid thickness, measured using EF-TEM and S/N data for the different thickness regions (Scale bar is $500 \mathrm{~nm}$ ). (b) High-resolution structure of AAV determined from particles in solution. Colored radial densities represent 5-nm slices through the interior of the EM map. Scale bar is $5 \mathrm{~nm}$. (c) A region of interest is shown at 5 seconds and 20 seconds time points (d) FSC plot (blue) indicates a structural resolution of $3.22-\AA$ according to the gold-standard criteria and the Cref(0.5) plot (red). (e) Density for an individual VP1 capsid protein from the liquid-phase structure determined with reference-free procedures. Computed density of the crystal structure at 3.2- $\AA$-resolution shows good agreement with the EM map. Scale bar is $10 \AA$. 